\title{
A Reconfigurable Cognitive Radio Antenna Design
}

M. Al-Husseini ${ }^{(1)}$, Youssef Tawk ${ }^{(2)}$, C. G. Christodoulou ${ }^{*(2)}$, K. Y. Kabalan ${ }^{(1)}$, A. El Hajj ${ }^{(1)}$

(1) ECE Department, American University of Beirut, Beirut 1107 2020, Lebanon

(2) ECE Department, University of New Mexico, Albuquerque, NM 87131, USA

E-mail: husseini@ieee.org

\section{Introduction}

According to the Federal Communications Commission (FCC), a cognitive radio is "a radio that can change its transmitter parameters based on interaction with the environment in which it operates" [1]. Thus, in cognitive radio system, we need the capability to sense the spectrum ("sensing" antenna), and communicate ("reconfigurable communicating" antenna).

Some research has been done related to the design of antennas for cognitive radio systems. In [2], a combination of wideband and narrowband antennas into the same volume is presented. The wideband antenna is a CPW-fed printed hourglass-shaped monopole that operates from 3 to $11 \mathrm{GHz}$. The narrowband antenna is a microstrip patch printed on the reverse side of the substrate, and connected to the wideband antenna via a shorting pin and designed to operate from 5.15 to $5.35 \mathrm{GHz}$. In [3], a frequency reconfigurable antenna to cover either the $3-5 \mathrm{GHz}$ or the $5-8 \mathrm{GHz}$ bands is designed. In the proposed structure, an UWB antenna is also incorporated, making it suitable for cognitive radio communication.

\section{Antenna System Configuration}

The presented cognitive radio antenna system is comprised of two microstrip-linefed monopoles printed on a $1.6 \mathrm{~mm}$-thick Taconic TLY substrate with a dielectric constant of 2.2. The two antennas share a common partial ground and are $20.5 \mathrm{~mm}$ apart, center to center. The configuration of the antenna system is detailed in Fig. 1.

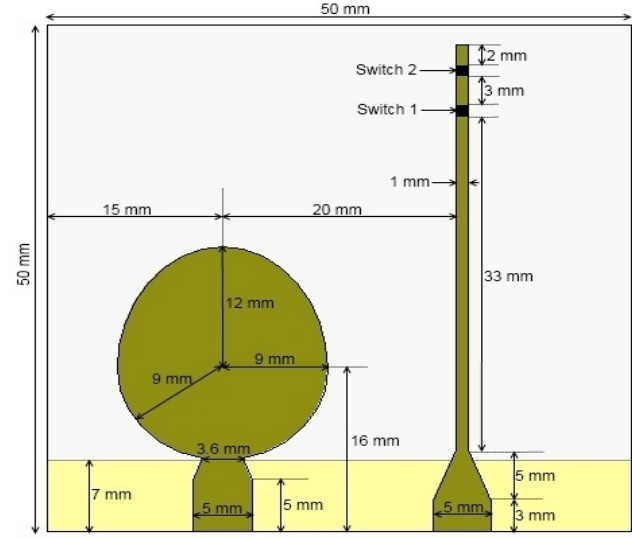

Fig.1 The antenna system structure

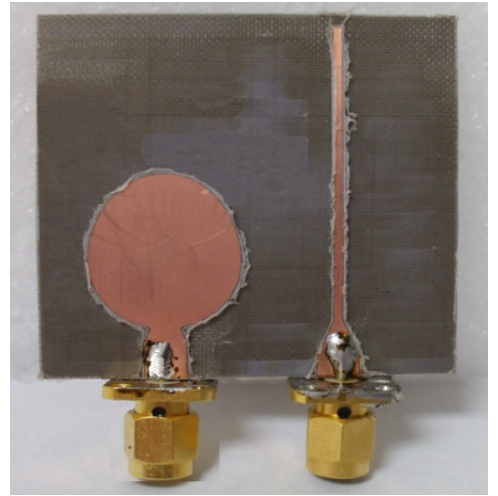

Fig.2 The fabricated prototype 
The sensing antenna is based on an egg-shaped patch, which is obtained by combining a circle and an ellipse at their centers [4]. The circle has a radius of $9 \mathrm{~mm}$, which is also the minor radius of the ellipse. The major radius of the ellipse is $12 \mathrm{~mm}$. A small tapered microstrip section matches the $50-\Omega$ feed to the input impedance of the patch. The design of the patch, the partial ground plane, and the feed matching section guarantee a UWB response of the sensing antenna.

The communicating antenna is a $40 \mathrm{~mm}$-long $1 \mathrm{~mm}$-wide microstrip line connected to a $50-\Omega$ feed line via a matching section. This structure yields multiple resonances in the 3.1-10.6 GHz UWB frequency range. Two $1 \times 1 \mathrm{~mm}^{2}$ electronic switches are incorporated along the antenna for use in a frequency reconfigurability scheme. By controlling these switches, the length of the antenna is changed, thus leading to various resonance frequencies. Additional switches can be used to obtain more resonances.

\section{Results and Discussion}

Here, three switching cases are considered: Case 1 where both switches are deactivated, Case 2 where Switch 1 is ON and Switch 2 is OFF, and Case 3 where both switches are activated. All cases were designed using HFSS v11. A prototype was fabricated and the return loss of the two antennas and their intercoupling was measured for the three switching cases. Without loss of accuracy, copper tapes were used in the prototype to represent the switches in their activated state. A photo of the fabricated prototype is shown in Fig. 2.

The measured and computed return loss plots of the sensing antenna, for Case 1, are shown in Fig. 3(a). Despite a little shift between them, an impedance bandwidth covering the UWB range is revealed. A similar UWB return loss is obtained in the two other cases. The computed pattern of the antenna at $5 \mathrm{GHz}$ is shown normalized in Fig. 3(b) for the H-plane (solid line) and the E-plane (dashed line). The pattern is omnidirectional, and this is guaranteed by the antenna's structure as a printed monopole over a partial ground plane. Similar results were obtained in the two other cases. At $5 \mathrm{GHz}$, the peak gain is about $6 \mathrm{~dB}$.

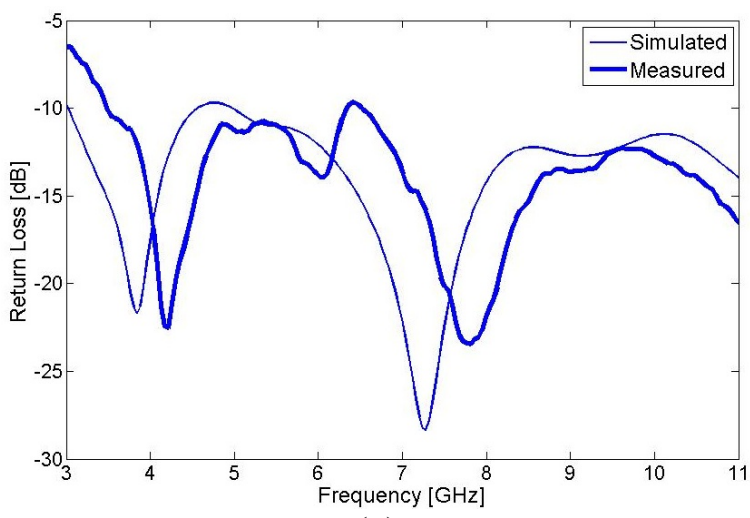

(a)

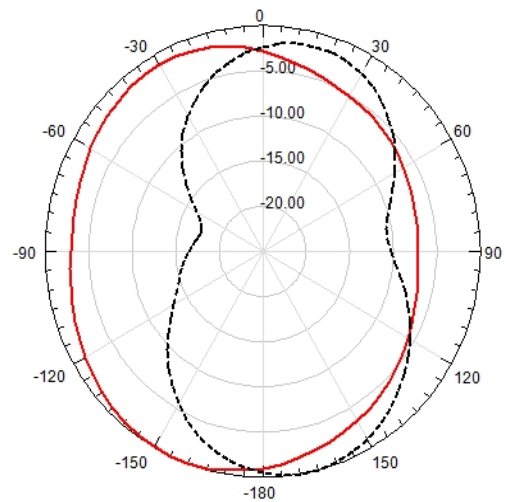

(b)

Fig.3 (a) Return loss of the sensing antenna, and (b) its radiation pattern at $5 \mathrm{GHz}$ 
Fig. 4 compares the measured and simulated return loss of the communicating antenna, for the 3 cases. Good agreement is witnessed in all cases. A superimposition of the measured return loss is given in Fig. 4(d), which shows clear frequency reconfigurability and a coverage of a large portion of the UWB range using only two switches.

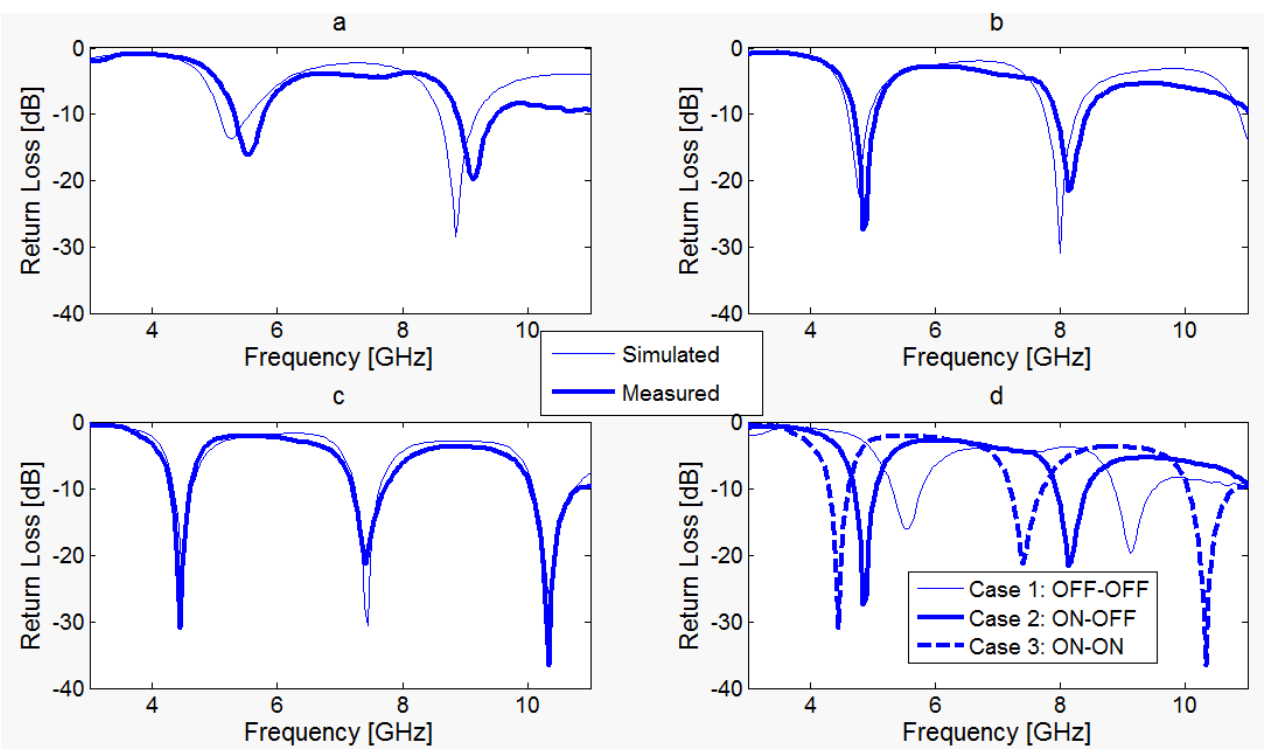

Fig.4 (a-c) Return loss plots of the communicating antenna for Cases 1-3 respectively, and (d) the superimposed measured plots for the 3 cases

The coupling between the two antennas for Case 3 is shown in Fig. 5. In all three cases, the coupling is less than $-10 \mathrm{~dB}$ for all frequencies. Specifically, the coupling figures at the communicating antenna's resonance frequencies are listed in Table 1, which shows the presented design offers well reduced coupling.

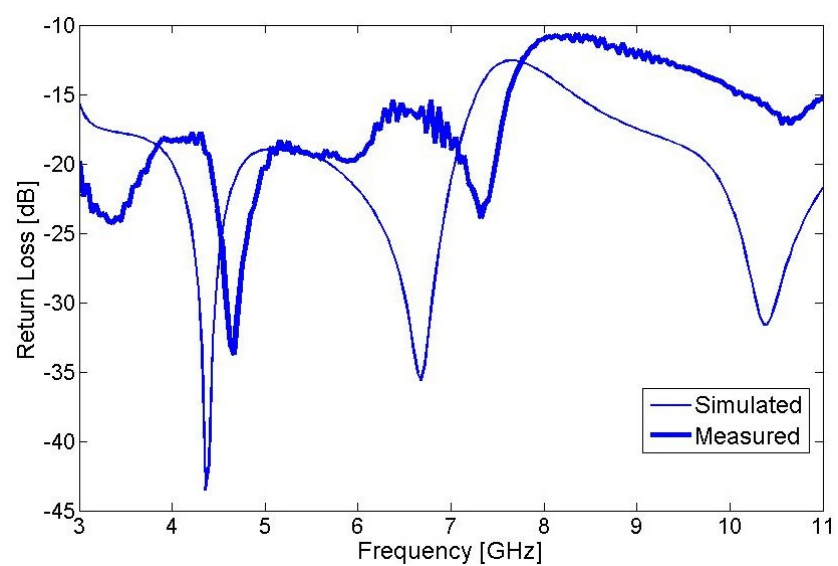

Fig. 5 Coupling between the two antennas for Case 3

Table1 Measured coupling at the resonance frequencies

\begin{tabular}{|l|c|c|c|c|c|c|c|}
\hline & \multicolumn{2}{|c|}{ Case 1 } & \multicolumn{2}{c|}{ Case 2 } & \multicolumn{3}{c|}{ Case 3 } \\
\hline f (GHz) & 5.55 & 9.15 & 4.85 & 8.15 & 4.44 & 7.41 & 10.33 \\
\hline S21 (dB) & -31.4 & -14.8 & -16.8 & -15.5 & -21.3 & -22.6 & -15.7 \\
\hline
\end{tabular}


Similar to the UWB antenna, the communicating antenna possesses omnidirectional patterns. The computed patterns are shown in Fig. 6 at the resonance frequencies for the three cases. The omnidirectional property degrades with increasing frequency, and as expected, sidelobes appear in the E-plane patterns at high frequencies. The peak gain values are given in Table 2 for the resonance frequencies.

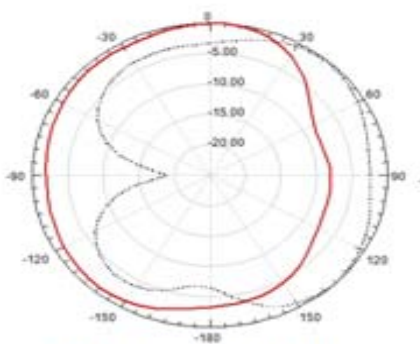

Case 1: $5.25 \mathrm{GHz}$

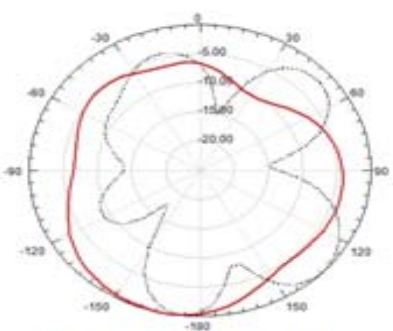

Case $1: 8.85 \mathrm{GHz}$

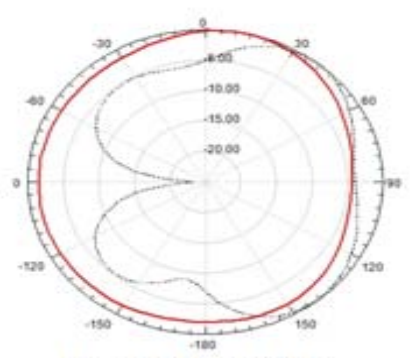

Case 2: $4.75 \mathrm{GHz}$

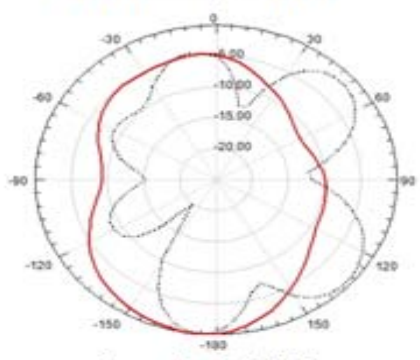

Case 2: $8.0 \mathrm{GHz}$

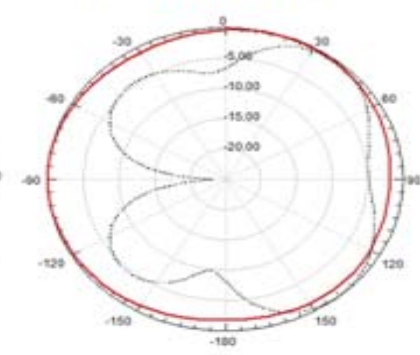

Case 3: $4.44 \mathrm{GHz}$

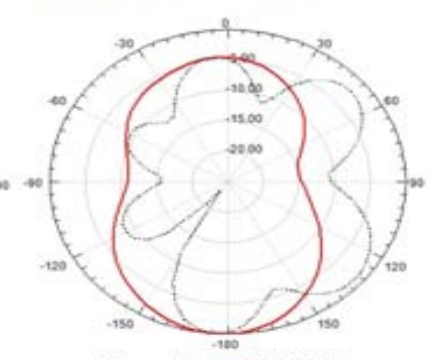

Case $3: 7.44 \mathrm{GHz}$

Fig.6 Patterns of the communicating antenna (dashed/solid line: E/H-plane)

Table 2 Computed peak gain at the resonance frequencies

\begin{tabular}{|l|c|c|c|c|c|c|c|}
\hline & \multicolumn{2}{|c|}{ Case 1 } & \multicolumn{2}{c|}{ Case 2 } & \multicolumn{3}{c|}{ Case 3 } \\
\hline f (GHz) & 5.25 & 8.85 & 4.75 & 8 & 4.44 & 7.44 & 10.35 \\
\hline Gain (dB) & 6.5 & 4.68 & 5.84 & 4.5 & 4.76 & 5.14 & 4.52 \\
\hline
\end{tabular}

\section{Conclusion}

This paper presents a new antenna design for cognitive radio communication. A prototype antenna was fabricated and tested. A good agreement was found between the simulated and measured data. A good coupling was achieved between the sensing and the communicating antenna.

\section{References}

[1] FCC, "Report of the spectrum efficiency working group", FCC spectrum policy task force, Tech. Rep., Nov. 2002.

[2] E. Ebrahimi, and P. S. Hall, "A dual port wide-narrowband antenna for cognitive radio", The third European Conference on Antennas and Propagation, pp. 809-812, Mar. 2009.

[3] Y. Tawk, and C. G. Christodoulou, "A New reconfigurable antenna for cognitive radio communication', IEEE Antennas and wireless propagation letters, Dec. 2009.

[4] M. Al-Husseini, A. Ramadan, Y. Tawk, A. El-Hajj, and K.Y. Kabalan, "Design and Ground Plane Consideration of a CPW-Fed UWB Antenna," The International Conference on Electrical and Electronics Engineering, pp.II-151-II-153, 5-8 Nov. 2009, Bursa, Turkey. 